\title{
The role of surgical management of BCG vaccine-induced regional suppurative lymphadenitis in children: a 7 years' experience from one medical center
}

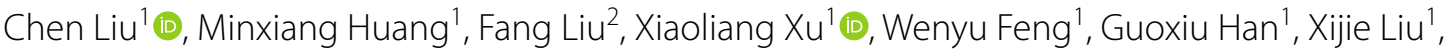 \\ Bufeng Zheng ${ }^{1}$, Lei Geng ${ }^{1^{*}}$ (1) and Tingliang Fu ${ }^{1}$ (D)
}

\begin{abstract}
Background: The management strategy of Bacille Calmette-Guérin (BCG) vaccine-induced regional suppurative lymphadenitis in children is still controversial and more clinical studies are needed. We therefore present a surgical case series to explore the role of surgical management for this dilemma.

Methods: From January 2013 to June 2020, data from 65 patients diagnosed with BCG vaccine-induced regional suppurative lymphadenitis were retrospectively reviewed. Clinical characteristics, ultrasonographic findings, surgical procedures, perioperative management, and outcome were analyzed. The association between postoperative seroma and symptom duration, skin involvement, and postoperative hospital stay were compared using Yates's corrected Chisquare test and Student's t-test for statistical analysis. The follow-up period ranged from three to six months.

Results: Of the 65 cases, the median age at presentation was 3.4 months. All patients were full-term with normal range of birth weight and received a BCG vaccination in the first $24 \mathrm{~h}$ of life. All patients underwent surgical excision of the abscess with the involved lymph node(s). Postoperative seroma formation was found in 20 patients and fine needle aspiration was needed. There was no significant association between postoperative seroma formation with symptom duration, skin involvement, and postoperative hospital stay. No oral anti-tubercular agents were given postoperatively. The mean length of postoperative hospital stay was $6.02 \pm 1.62$ days. Sixty-four cases $(98.46 \%)$ received only one procedure and recovered. One patient required a second procedure due to postoperative sinus.

Conclusions: The present study showed that surgical excision of the abscess with involved lymph node(s) is one of the choices for BCG vaccine-induced suppurative lymphadenitis, but special attention should be paid to controlling the surgical indications. Intraoperative meticulous manipulation and postoperative care are crucial to achieve a good outcome.
\end{abstract}

Keywords: Bacille Calmette-Guérin vaccine, Adverse reaction, Regional suppurative lymphadenitis, Surgical management, Children

*Correspondence: 38181141@qq.com

1 Department of Pediatric Surgery, Binzhou Medical University Hospital,

Shandong 256603, China

Full list of author information is available at the end of the article

\section{Background}

Mycobacterium bovis Bacille Calmette-Guérin (BCG) strain, a live attenuated vaccine, has been widely vaccinated to prevent tuberculosis, which can reduce the risk of pulmonary tuberculosis by over $50 \%$ 
[1-3]. However, the incidence of BCG vaccine-induced adverse reactions may reach 5 cases per 1000 doses [4-6]. BCG vaccine-induced infections can resolve without any intervention in parts of cases [7], but some adverse reactions, especially suppurative lymphadenitis with perforation and chronic sinus formation need a long time to manage and may cause parental distress [3]. Different surgical treatment strategies for BCG vaccine-induced regional suppurative lymphadenitis, including needle aspiration with or without local instillation of isoniazid, open drainage of the abscess (not recommended), excochleation, and node excision have been introduced, but the effectiveness is still unclear [8-10]. We present seven years' experience and explore the role of surgical treatment for BCG vaccine-induced regional suppurative lymphadenitis at a university medical center.

\section{Methods}

From January 2013 to June 2020, sixty-five patients who received intradermal vaccination of BCG vaccine $[0.1 \mathrm{ml}$, China's BCG strain (BCG D2 PB302SII A 10 strain) derived from Denmark in the 1950s] [11] in the deltoid region of the left upper arm on the first day of life and suffered ipsilateral regional lymphadenitis were admitted to a university medical center. The inclusion criteria of participants in the present study were as follows: (1) medical history of BCG vaccination; (2) up to 5 years of age; (3) presenting a painless mass in left axillary and/or supraclavicular, chest wall, scapular, neck corresponding to the BCG vaccination site, with reddish skin, with fluctuant, sinus formation or rapidly enlarged lymph node(s); (4) no immunodeficiency status, no generalized lymphadenopathy. Exclusion criteria of the participants included (1) age less than or equal to 1 month; (2) mass size less than $2 \mathrm{~cm}$ without overlying skin infiltration, fluctuant or sinus formation; (3) nonspecific lymphadenitis; (4) with other conditions who did not tolerate general anesthesia. All patients' guardians were informed regarding the details of the surgical procedure, the risk of general anesthesia, and associated complications. Age in months, gender, location and size of the mass, chest $\mathrm{X}$ ray (CXR), sonographic findings, pathological findings, postoperative seroma formation, and length of postoperative hospital stay were retrospectively collected. The mass sizes of the resected specimens were measured. The association between postoperative seroma formation and symptom duration, skin involvement, and postoperative hospital stay were analyzed using IBM SPSS Statistics software version 25.0 (IBM Corp., Armonk, NY, USA). Yates's corrected Chi-square test and Student's t-test were used for statistical analysis.

\section{Indications for surgical procedure}

Based on the literature review $[3,10]$ and our experience, in the present study, the surgical indications for abscess with involved lymph nodes excision were as follows: the lesion affecting overlying skin, spontaneously perforated or sinus formation, fixed lymph nodes, the mass size greater than or equal to $2 \mathrm{~cm}$, developed and enlarged rapidly in two months or liquefaction feature revealed by ultrasonography, or mass lasting for three to six months without regression.

\section{Preoperative management}

Clinical observations of the symptoms, signs, and progress/regression of the lesion of BCG lymphadenitis were consulted by pediatricians. Indications for surgical management were assessed by pediatric surgeons. No anti-tubercular agents were given before surgery. Fine needle aspiration of the abscess was performed if the larger abscess had significant fluctuations or the abscess was intended to perforate spontaneously due to the thinner involved overlying skin. As one of the surgical steps, a larger abscess should be aspirated before surgical excision to reduce the risk of intraoperative rupture and contamination of the wound. The pus collection was stained and cultured for acid fast bacilli (AFB).

\section{Surgical procedures and postoperative management}

Due to the liquefied area or involved skin, an elliptical incision parallel to the skin crease was chosen. The subcutaneous abscess with involved lymph node(s) located under the deep fascia plane were excised. The blood vessels from the involved lymph node(s) were ligated using 5-0 absorbable suture. After removal of the mass, the wound cavity was flushed with isoniazid injection $(50 \mathrm{mg}$ in $5 \mathrm{ml}$ sterilized normal saline), according to the literature $[12,13]$. Then the subcutaneous tissue and skin incision were separately sutured by $5-0$ absorbable suture. No drainage was placed in the wound. Appropriate pressure dressing of the wound site was maintained for $48 \mathrm{~h}$. Dressing change was conducted on the second postoperative day. The skin sutures were removed on the ninth to tenth postoperative day. Histopathological examination of excised samples was carried out.

Wound healing status (first intention), no evidence of seroma formation or infection, or no palapable enlarged lymph node in two postoperative weeks were used as clinical criteria of complete remission.

\section{Follow-up}

All children' parents or guardians were instructed to follow-up with their children in the clinic or by telephone interview within two weeks after operation. Then, 
according to the patients' status, the follow-up period ranged from three to six months postoperatively. Incisional wound scarring, any evidence of abscess, sinus formation, or enlarged lymph nodes in the operative site were observed.

\section{Ethics considerations}

Informed consent was obtained from the parents/legal guardian(s) of all children involved in the study. The study was approved by the Ethics Committee of Binzhou Medical University Hospital (LW-013).

\section{Results}

A total of 65 cases had a median age at symptom onset of 3.4 (IQR: 2.38-5.1) months. Table 1 lists the demographic and clinical characteristics. All patients had regional lymphadenitis corresponding to the BCG vaccination site. Overall, 87 (92\%) children having regional involved lymph node(s) were located in the left axillary (Fig. 1) in

Table 1 Demographic and clinical characteristics of all participants at admission

\begin{tabular}{|c|c|}
\hline \multicolumn{2}{|l|}{ Characteristics } \\
\hline Number of participants & 65 \\
\hline $\begin{array}{l}\text { Median age (IQR) } \\
\text { Age group (months), } n(\%)\end{array}$ & $3.4(2.38-5.1)$ \\
\hline$>1-4$ & $42(64.62)$ \\
\hline $4-8$ & $14(21.54)$ \\
\hline $8-12$ & $4(6.15)$ \\
\hline$>12$ & $5(7.69)$ \\
\hline \multicolumn{2}{|l|}{ Gender, $n(\%)$} \\
\hline Male & $55(84.62)$ \\
\hline Female & $10(15.38)$ \\
\hline The male-to-female ratio & 5.5: 1 \\
\hline \multicolumn{2}{|c|}{ Duration of symptom (months), $n$ (\%) } \\
\hline$\leq 3$ & $60(92.31)$ \\
\hline$>3$ & $5(7.69)$ \\
\hline \multicolumn{2}{|l|}{ Location of lymphadenitis, $n$ (\%) } \\
\hline Left axillary & $59(90.77)$ \\
\hline Left supraclavicular & $3(4.62)$ \\
\hline Left neck & $1(1.54)$ \\
\hline Left axillary and supraclavicular & $1(1.54)$ \\
\hline Left axillary and scapular & $1(1.54)$ \\
\hline \multicolumn{2}{|l|}{ Presentation, $n(\%)$} \\
\hline Yes & $49(75.38)$ \\
\hline No & $16(24.62)$ \\
\hline Yes & $3(4.62)$ \\
\hline No & $62(95.38)$ \\
\hline \multicolumn{2}{|l|}{ Mass size $(\mathrm{cm}), n(\%)$} \\
\hline $2-3$ & $8(12.31)$ \\
\hline$\geq 3$ & $57(87.69)$ \\
\hline
\end{tabular}

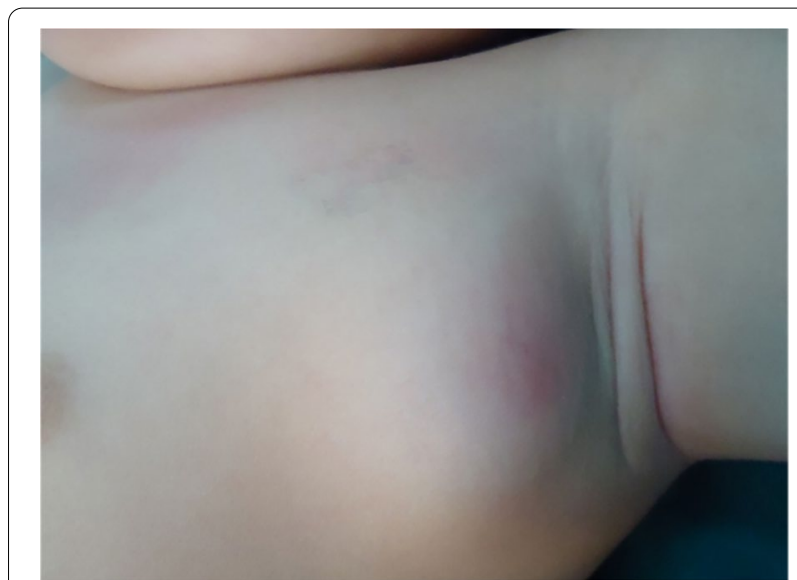

Fig. 1 Appearance of the mass with skin involvement in the left axillary and chest wall

59 cases $(90.77 \%)$. All patients had no fever, night sweats, emaciation, or other systemic symptoms.

Chest $\mathrm{X}$ ray showed no pulmonary lesions in all patients. A lumpy high-density shadow was found in the left armpit in eight cases (12.3\%). Ultrasonography showed an enlarged lymph node(s) with liquefaction area indicating abscess formation in most cases. The AFB cultures were negative in all cases. Specimen measurements showed that the mass size ranged 2 to $5 \mathrm{~cm}$ and $87.69 \%$ was greater than or equalled to $3 \mathrm{~cm}$. Pathological findings showed that a typical tuberculous granulomatous lesion with abscess formation (Fig. 2). The existence of epithelioid cells, multinucleated giant cells, and lymphocytic infiltration in the involved lymph nodes were consistent with BCG vaccine-induced suppurative lymphadenitis.

On the second postoperative day, seroma formation was found in 20 patients (30.77\%). Table 2 showed that there was no significant association between postoperative seroma formation with symptom duration, skin involvement, and the length of postoperative hospital stay. After fine needle aspiration (ranging 1-6 times) and proper pressure dressing were conducted, the collection reduced gradually and disappeared completely about a week. The mean length of postoperative hospital stay was $6.02 \pm 1.62$ days. The incision healed by first intention in all cases. One patient presented an occurrence of sinus formation with intermittent purulent discharge at two weeks after the first procedure. Frequent dressing changes were needed. The patient received oral antitubercular treatment for 3 months which had no effect on wound healing. One year later, the patient underwent a second procedure to remove the residual involved lymph nodes and sinus. The pathological findings showed the affected lymph nodes' size was $2 \mathrm{~cm}$ times $0.9 \mathrm{~cm}$.

IQR interquartile range 


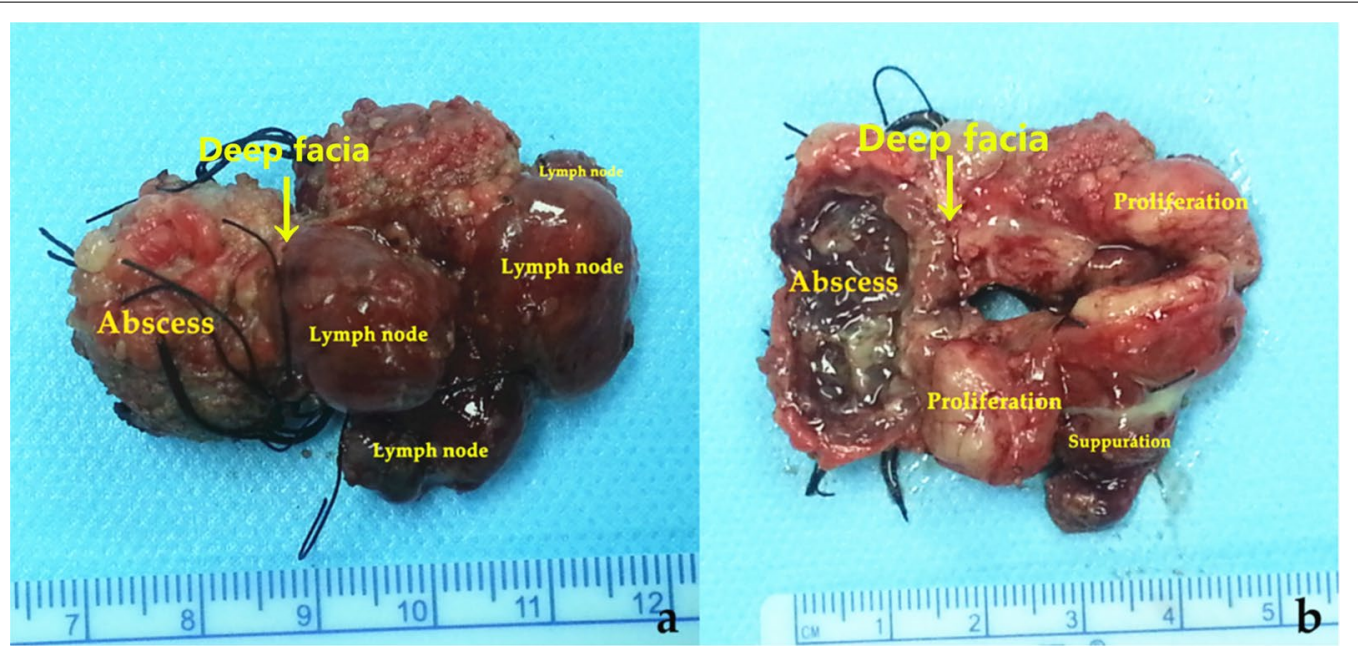

Fig. 2 Gross appearance of the excised specimen. a Enlarged lymph nodes with abscess formation. b Subcutaneous abscess, proliferative and suppurating lymph nodes (in sectional view)

Table 2 Statistical analysis of the comparison of variables between seroma and no seroma formation groups

\begin{tabular}{|c|c|c|c|c|c|c|c|c|c|}
\hline \multirow[t]{2}{*}{ Variables } & \multirow{2}{*}{$\begin{array}{l}\begin{array}{l}\text { Overall } \\
n=65\end{array} \\
\text { Mean } \pm \text { SD }\end{array}$} & \multicolumn{3}{|c|}{$\begin{array}{l}\text { No seroma formation } \\
n=45\end{array}$} & \multicolumn{3}{|c|}{$\begin{array}{l}\text { Seroma formation } \\
n=20\end{array}$} & \multirow[t]{2}{*}{$95 \% \mathrm{Cl}$} & \multirow[t]{2}{*}{$P$-value } \\
\hline & & Mean \pm SD & Range & $n$ & Mean \pm SD & Range & $n$ & & \\
\hline Symptom duration (months) & $1.90 \pm 5.12$ & $2.03 \pm 5.57$ & $0.03 *-36$ & & $1.63 \pm 4.18$ & $0.03 *-19$ & & $-3.18-2.39$ & $0.778^{\#}$ \\
\hline Involved skin & & & & 33 & & & 16 & & $0.792^{*}$ \\
\hline Postoperative hospital stay (days) & $6.02 \pm 1.62$ & $5.91 \pm 1.66$ & $3-9$ & & $6.25 \pm 1.59$ & $4-9$ & & $-0.54-1.22$ & $0.445^{\#}$ \\
\hline
\end{tabular}

SD standard derivation; * ${ }^{*}$ larger mass was incidentally found by guardians in some cases; ${ }^{\text {} S t u d e n t ' s ~ t-t e s t ; ~}{ }^{*}$ Yates's corrected Chi-square test

The patient recovered uneventfully. The follow-up period lasted for six months and no enlarged lymph nodes were found with good cosmetic appearance.

\section{Discussion}

BCG vaccine is a live attenuated strain which is widely used for preventing tuberculosis $[3,8]$. The adverse reactions, including abscess or ulceration of the injection site, regional lymphadenitis may cause parental distress [2]. BCG vaccine-induced lymphadenitis, defined as the development of ipsilateral regional enlarged lymph node(s), is considered as the most common severe complication [14, 15]. In our data, most cases occurred in male infants and within 6 months of age, it is consistent with the literature [3].

The natural course of suppurative lymphadenitis is perforation and chronic sinus formation, which may cause parental stress or increase the risk of spread [2]. BCG vaccine-induced lymphadenitis is classified into suppurative and non-suppurative forms [7]. More than half of non-suppurative lymphadenitis will progress to the suppurative form which presents swelling and redness of the skin, fluctuation, rupture spontaneously and chronic sinus formation [9, 16]. Different treatment strategies for lymphadenitis induced by BCG vaccination have been reported, which include "watch and see" with regular follow-up [4, 7], anti-tubercular treatment [3, 12], needle aspiration with or without isoniazid instillation $[4,12,17]$, incision and drainage [17], excochleation of the necrotic tissue [10], or surgical excision of involved lymph nodes [18-22]. However, there are no definite treatment guidelines proposed for BCG vaccine-induced suppurative lymphadenitis [16, 23]. Needle aspiration is an effective measure in cases with suppurative lymphadenitis, but in those with larger size, multiple, and matted lymph nodes, needle aspiration usually has no proven efficacy [17]. Thus, surgical removal of the involved lymph nodes and abscess to shorten the length of healing and to avoid sinus formation was proposed by several authors $[8,14,16,17]$.

Surgical indications for the management of BCG vaccine-induced regional suppurative lymphadenitis need to be defined so far $[17,23]$. Based on the literature review and our experience, hereby, we propose the indications 


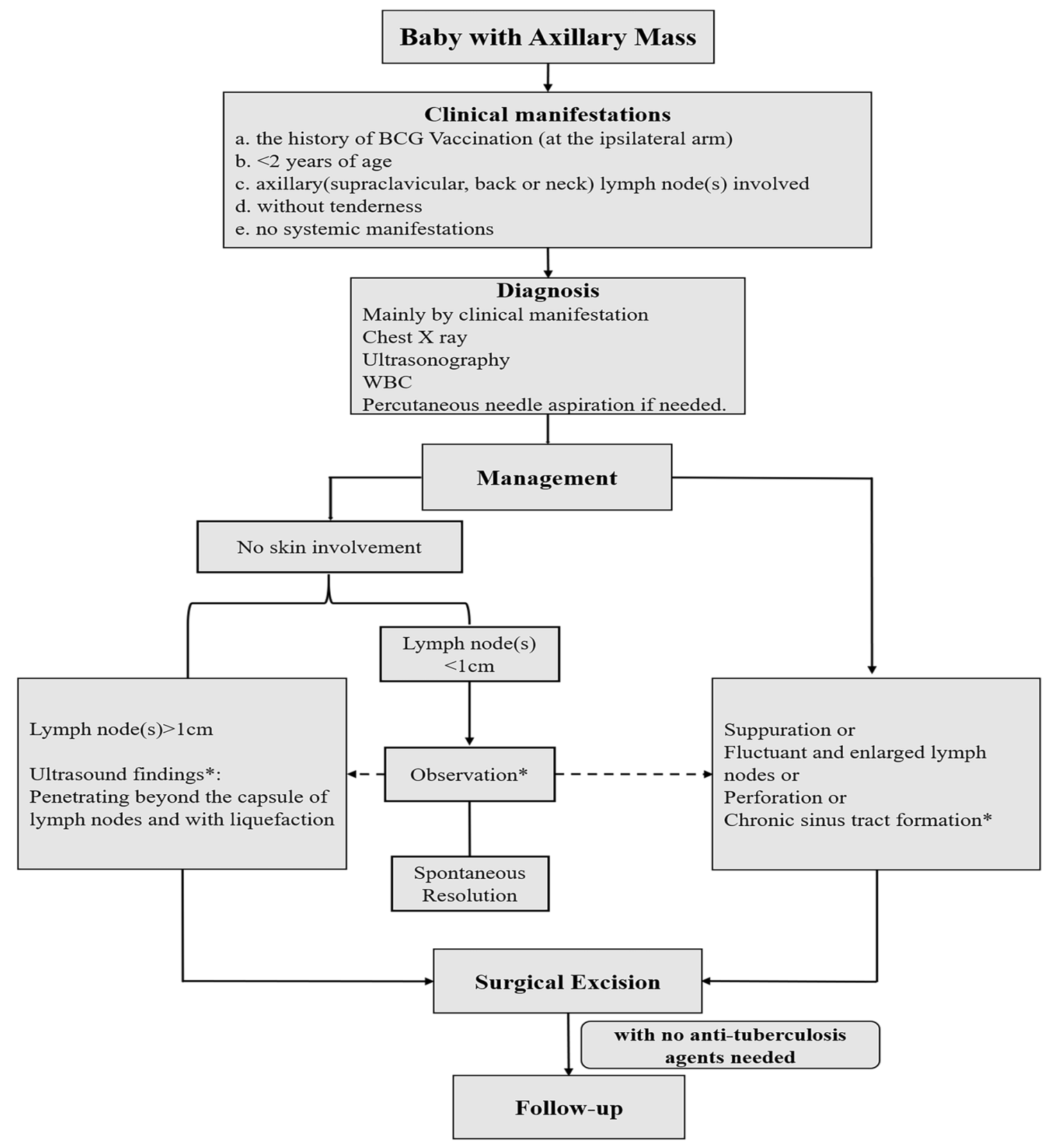

Fig. 3 Flow chart for the management of BCG vaccine-induced regional lymphadenitis

for surgical management as follows: the enlarged lymph node greater than $1 \mathrm{~cm}$ in size [9] and ultrasonography revealing liquefaction and capsule penetration of the lymph node(s); rapidly enlarging or infiltrating nodes [22]; infiltrating overlying skin [10, 21, 22]; spontaneously perforated or sinus formation [10, 19, 21]; fixation of regional lymph node(s) [14]; lymph nodes larger than $1.5-3 \mathrm{~cm}[10,19,21]$; mass lasting for 3 to 6 months without regression $[15,19]$; or repeated collections after needle aspiration, especially with matted and multilocular nodes [17, 24].

Although the surgical management of BCG vaccineinduced suppurative lymphadenitis has been reported with good results $[9,18,25]$, the disadvantages are the exposure of sedation or general anesthesia, potential intra- and post-operative complications [3, 17], and reoccurrence due to residual adenopathy with sinus formation requiring more than one procedure [26]. Nevertheless, 
surgical excision, as an invasive technique, still needs to balance the risks of general anesthesia and potential surgical complications. Since the healing or resolving period of needle aspiration usually needs 2 to 6 months [20, 24] and about half of the patients face the risk of chronic sinus formation and need removal of affected lymph nodes [18], surgical excision of the abscessed lymph nodes with involved skin and subcutaneous abscess was a choice to deal with this disorder [10, 27]. In our case series, local skin was not involved in 16 cases, but liquefication or capsule penetration of the involved lymph nodes was found by ultrasonography, which indicated that the lesion was intended to infiltrate the subcutaneous tissue and overlying skin [9]. Early operative intervention should be considered an option [14].

However, surgeons should pay more attention to the delicate surgical procedures. (1) Large abscess should be aspirated before excision to avoid intraoperative rupture and contamination of the wound space; (2) removal of the subcutaneous abscess by using sharp dissection in an appropriate plane instead of electrocautery use was strongly advised to avoid wound dehiscence. (3) The excisional extent should be strictly limited to the involved nodes to protect the axillary structures, such as vessels, nerves, and normal lymph nodes from damage; (4) if the lesions tightly adhere the axillary sheath, electrocautery should not be used for dissection because of its underlying damage to the axillary structures [19]. In addition, before wound closure, fluid and air in the wound space should be squeezed out. An appropriate pressure dressing of the wound site was conducted to reduce the occurrence of postoperative seroma.

In our case series, postoperative seroma formation occurred in nearly one-third of patients. The association of postoperative seroma formation and duration of symptoms is unclear. In a study, patients received macrolide therapy for 10 days before surgery to reduce tissue edema, which may make the surgical dissection safer and easier [19]. Patients with postoperative seroma formation underwent serial fine needle aspiration. A pressure dressing over the wound site was vital.

Anti-tubercular treatment is not advised for BCG vaccine-induced regional lymphadenitis $[9,10,19]$. However, some reports revealed that needle aspiration with a single dose of 50-mg isoniazid instillation may shorten the healing period by 1 month [12]. Wu et al. [13] reported AFB positive rate of pus smears is $18 / 58(31.03 \%)$ and local use of isoniazid was recommended to eliminate residual Mycobacterium bovis. Due to potential contamination by mycobacterium bovis from intraoperative abscess leakage, a single dose of isoniazid injection diluted with normal saline was used to flush the wound space before wound closure.
Based on the literature $[4,10,12,14,18,19,27-30]$ and our preliminary experience, a flow chart (Fig. 3) was introduced for the management of BCG vaccine-induced regional lymphadenitis.

Our study has several limitations. First, it is a retrospective study with a relatively small sample size and all cases were from one region and treated in a single medical center. Therefore, these results may not be generalized to other regions. Second, the safety and time-saving effects of surgical management on the clinical evolution of BCG vaccine-induced suppurative lymphadenitis need to be studied.

\section{Conclusions}

There was a substantial variety in the management of BCG vaccine-induced suppurative lymphadenitis, so the optimal approach remains to be clarified. The present observation showed that surgical excision of the subcutaneous abscess with involved lymph node(s) is one of the choices, but special attention should be paid to controlling the indications for surgical management. Intraoperative meticulous manipulation and postoperative care are crucial to achieve a good outcome.

\section{Abbreviations \\ BCG: Bacille Calmette-Guérin; AFB: Acid fast bacilli; CXR: Chest X ray; SD: Standard deviation.}

\section{Acknowledgements}

We thank all patients and their families who participated in this study and acknowledge the assistance of colleagues in the Department of Pediatric Surgery, Binzhou Medical University Hospital, Binzhou, Shandong Province, China.

\section{Authors' contributions}

$\mathrm{CL}$ and TLF wrote the main manuscript text. GL, FL, and TLF supervised and designed the study. MXH, XLX, WYF, GXH, XJL, and BFZ collected and analyzed the data. All authors read and approved the final manuscript.

Funding

This research received no external funding.

\section{Availability of data and materials}

The data presented in this study are available on request from the corresponding author. Email should be sent to 38181141@qq.com.

\section{Declarations}

\section{Ethics approval and consent to participate}

Informed consent was obtained from the parents/legal guardian(s) of all children involved in the study. All methods of the study protocol were approved by the Ethics Committee of Binzhou Medical University Hospital in accordance with the Declaration of Helsinki.

Consent for publication

Not applicable.

\section{Competing interests}

The authors declare no competing interests. 


\section{Author details}

${ }^{1}$ Department of Pediatric Surgery, Binzhou Medical University Hospital, Shandong 256603, China. ${ }^{2}$ Child Health Section, Binzhou Medical University Hospital, Shandong 256603, China.

Received: 4 May 2021 Accepted: 3 August 2021

Published online: 11 August 2021

\section{References}

1. Lotte A, Wasz-Höckert O, Poisson N, Dumitrescu N, Verron M, Couvet E. BCG complications. Estimates of the risks among vaccinated subjects and statistical analysis of their main characteristics. Adv Tuberc Res. 1984;21:107-93.

2. Milstien JB, Gibson JJ. Quality control of BCG vaccine by WHO: a review of factors that may influence vaccine effectiveness and safety. Bull World Health Organ. 1990;68(1):93-108. https://doi.org/10.1146/annurev.publh ealth.11.1.127.

3. Cuello-Garcia CA, Perez-Gaxiola G, Jimenez Gutierrez C. Treating BCG-induced disease in children. Cochrane Database Syst Rev. 2013;1:CD008300. https://doi.org/10.1002/14651858.CD008300.pub2.

4. Goraya JS, Virdi VS. Bacille Calmette-Guerin lymphadenitis. Postgrad Med J. 2002;78(920):327-9. https://doi.org/10.1136/pmj.78.920.327.

5. Hesseling AC, Rabie H, Marais BJ, Manders M, Lips M, Schaaf HS, et al. Bacille Calmette-Guerin vaccine-induced disease in HIV-infected and HIVuninfected children. Clin Infect Dis. 2006;42(4):548-58. https://doi.org/10. 1086/499953.

6. Holliday T, Seddon J, Whittaker E. G157(P) Adverse events in infants following bcg vaccination: preliminary results. Arch Dis Child. 2019;104(Suppl 2):A64. https://doi.org/10.1136/archdischild-2019-rcpch. 153.

7. Singla A, Singh S, Goraya JS, Radhika S, Sharma M. The natural course of nonsuppurative Calmette-Guerin bacillus lymphadenitis. Pediatr Infect Dis J. 2002;21(5):446-8. https://doi.org/10.1097/00006454-20020 5000-00026.

8. Venkataraman A, Yusuff M, Liebeschuetz S, Riddell A, Prendergast AJ. Management and outcome of Bacille Calmette-Guerin vaccine adverse reactions. Vaccine. 2015;33(41):5470-4. https://doi.org/10.1016/j.vaccine. 2015.07.103.

9. Baki A, Oncu M, Usta S, Yildiz K, Karaguzel A. Therapy of regional lymphadenitis following BCG vaccination. Infection. 1991;19(6):414-6. https://doi. org/10.1007/BF01726452.

10. Hengster P, Sölder B, Fille M, Menardi G. Surgical treatment of bacillus Calmette Guérin lymphadenitis. World J Surg. 1997;21(5):520-3. https:// doi.org/10.1007/pl00012279.

11. Zhao AH, Wang GZ, Fu LL, Xu M. Comparison of BCG vaccine quality standards of domestic and foreign. Chin J Antituberc. 2018;40(10):1125-8. https://doi.org/10.3969/j.issn.1000-6621.2018.10.017.

12. Noah PK, Pande D, Johnson B, Ashley D. Evaluation of oral erythromycin and local isoniazid instillation therapy in infants with Bacillus CalmetteGuerin lymphadenitis and abscesses. Pediatr Infect Dis J. 1993;12(2):1369. https://doi.org/10.1097/00006454-199302000-00006.

13. Wu W, Li B, Ni X, An Y. Clinic and curative effect analysis on 112 cases with strong reactions of lymph node caused by neonatal BCG vaccination. Chin J Antituberc, 2012; 34(4):241-244.https://d.wanfangdata.com.cn/ periodical/ChIQZXJpb2RpY2FsQ0hJTmV3UzlwMjEwNjE2Eg96Z2Zse noyMDEyMDQwMTAaCHJsZTdhNmVq

14. Engelis A, Kakar M, Meikšāns R, Petersons A. BCG-SSI $\left({ }^{\circledR}\right)$ vaccine-associated lymphadenitis: Incidence and management. Medicina (Kaunas). 2016;52(3):187-91. https://doi.org/10.1016/j.medici.2016.05.001.
15. Waili BA, Mufarajii NA, Hashmi SA, Ajmi AA, Sukaiti NA. Bacillus CalmetteGuerin vaccine-related complications in children in Oman. Ann Saudi Med. 2021:41(1):24-30. https://doi.org/10.5144/0256-4947.2021.24.

16. Daei Parizi M, Kardoust Parizi A, Izadipour S. Evaluating clinical course of BCG lymphadenitis and factors affect on it during a 5-year period in Kerman. Iran J Trop Pediatr. 2014;60(2):148-53. https://doi.org/10.1093/ tropej/fmt100.

17. Naumeri F, Khan MS, Rauf A, Imran S, Tahir M. Role of needle aspiration and surgical excision in management of suppurative Bacillus Calmette Guerin lymphadenitis. J Pak Med Assoc. 2018;68(7):1120-3 (PMID: 30317317).

18. Nazir Z, Qazi SH. Bacillus Calmette-Guerin (BCG) lymphadenitis-changing trends and management. J Ayub Med Coll Abbottabad. 2005;17(4):16-8 (PMID: 16599027).

19. Hassan RH, Aly AE, Kandil SM, Zaki M. Post-Bacillus Calmette-Guérin lymphadenitis in Egyptian children: an outbreak. Annals of Pediatric Surgery. 2012;8(3):69-73. https://doi.org/10.1097/01.XPS.0000413172.23876.7d.

20. Caglayan S, Arikan A, Yaprak I, Aksoz K, Kansoy S. Management of suppuration in regional lymph nodes secondary to BCG vaccination. Acta Paediatr Jpn. 1991;33(6):699-702. https://doi.org/10.1111/j.1442-200x. 1991.tb02593.x

21. Caglayan S, Yegin O, Kayran K, Timocin N, Kasirga E, Gun M. Is medical therapy effective for regional lymphadenitis following BCG vaccination? Am J Dis Child. 1987;141(11):1213-4. https://doi.org/10.1001/archpedi. 1987.04460110083029.

22. Nicol M, Eley B, Kibel M, Hussey G. Intradermal BCG vaccination-adverse reactions and their management. S Afr Med J. 2002;92(1):39-42. https:// doi.org/10.1016/S0248-8663(01)00522-7.

23. Baek SO, Ko HS, Han HH. BCG vaccination-induced suppurative lymphadenitis: four signs to pay attention to. Int Wound J. 2017;14(6):1385-7. https://doi.org/10.1111/iwj.12755.

24. Banani SA, Alborzi A. Needle aspiration for suppurative post-BCG adenitis. Arch Dis Child. 1994;71(5):446-7. https://doi.org/10.1136/adc.71.5.446.

25. Tam PK, Stroebel AB, Saing H, Lau JT, Ong GB. Caseating regional lymphadenitis complicating BCG vaccination: a report of 6 cases. Arch Dis Child. 1982;57(12):952-4. https://doi.org/10.1136/adc.57.12.952.

26. Bolger T, O'Connell M, Menon A, Butler K. Complications associated with the bacille Calmette-Guérin vaccination in Ireland. Arch Dis Child. 2006;91(7):594-7. https://doi.org/10.1136/adc.2005.078972.

27. Elsidig N, Alshahrani D, Alshehri M, Alzahrani M, Alhajjar S, Aljummah S, et al. Bacillus Calmette-Guerin vaccine related lymphadenitis in children: Management guidelines endorsed by the Saudi Pediatric Infectious Diseases Society (SPIDS). Int J Pediatr Adolesc Med. 2015;2(2):89-95. https:// doi.org/10.1016/j.ijpam.2015.05.003.

28. Rermruay R, Rungmaitree S, Chatpornvorarux S, Brukesawan C, Wittawatmongkol O, Lapphra K, et al. Clinical features and outcomes of Bacille Calmette-Guérin (BCG)-induced diseases following neonatal BCG Tokyo-172 strain immunization. Vaccine. 2018;36(28):4046-53. https://doi. org/10.1016/j.vaccine.2018.05.098.

29. Riordan A, Cole T, Broomfield C. Fifteen-minute consultation: Bacillus Calmette-Guérin abscess and lymphadenitis. Arch Dis Child Educ Pract Ed. 2014;99(3):87-9. https://doi.org/10.1136/archdischild-2013-304457.

30. Jain A, Gupta S, Gupta P, Rohilla M, Gupta N, Srinivasan R, Rajwanshi A, Dey P. Fine-needle aspiration cytology of Bacille Calmette-Guerin adenitis: a perplexing distinct clinical entity. Diagn Cytopathol. 2021;49(2):22631. https://doi.org/10.1002/dc.24613.

\section{Publisher's Note}

Springer Nature remains neutral with regard to jurisdictional claims in published maps and institutional affiliations. 